\title{
Less than full analgesia can leave significant pain under-treated
}

$\mathrm{T}$ he IACUC made a poor decision in this scenario. The decision may not be compliant with regulations and needlessly puts the monkeys at risk of pain and suffering beyond that necessary to obtain quality data.

Given the challenges of accurately diagnosing minute-by-minute pain in monkeys, and the reality that the monkeys cannot self-medicate, all should err on the side of presuming pain and maximizing safe use of analgesics. All available guidance presumes leadership and judgment of veterinarians, who should be current in their field and actively involved with the animals. This standard of care to treat preemptively with multimodal analgesia is not isolated to GEU's veterinarians-it is included in the Guide and in guidance from professional veterinary groups ${ }^{1-4}$. IACUCs follow a principle by which we assume what is painful to humans may be painful to animals; a corollary is that effective pain management for humans may be a helpful guide for animal pain management. The standard of care for comparable major survival surgeries in humans includes multimodal pain management strategies, not just intermittent boluses of mid-potency opioids 5 .

True, the Animal Welfare Act does not specify that investigators must follow the veterinarian's consultation ${ }^{6}$. But the committee has approved a lower level of pain management than current veterinary standards promote. This puts the monkeys at risk of more than minor pain, with no scientific justification for compromising their welfare. It may violate the "adequate post-procedural care" provision of mandated adequate veterinary care, and raises questions about the investigator's training to provide proper post-procedure care and proper use of analgesics. If meeting minutes accurately reflect the consultation and deliberations, the USDA inspector should scrutinize this issue closely. The approved practice would not put the animals into Column E of the annual report, as that is only appropriate when the analgesics are suspected to interfere with data-not when IACUC and investigators allow what looks like under-treatment of pain for other reasons.

Faced with the investigator's vague assertions about how the animals look and without veterinary evaluation of the animals (at least, not in this scenario), the committee could mitigate its bad decision by requiring that the veterinarians and investigator develop a proper pain scoring system and compare multimodal analgesia to buprenorphineonly analgesia. Published clinical pain management data on monkeys are scarce. The GEU people could quantify measures such as time to reach for treats where monkeys must work for them, food and water consumption, postures and facial expressions. Human presence can affect these outcomes, so plans for video-monitoring would be necessary. The IACUC needs to hear more than that the animals "looked good."

I am also concerned about the IACUC's relationship with the veterinary team as described here, and why the committee would side with an investigator's veterinary judgment over standard veterinary practice and the on-site veterinarians' recommendations. Have the vets failed to properly educate the IACUC and researchers on the complexities of animal pain recognition, prevention and treatment? Are the veterinarians not forcefully advocating for best possible animal welfare? Does the IACUC have other reasons to dismiss GEU's veterinarians' judgment, and if so, should they ask the institutional officer to engage in some sort of review of the vet team's qualifications and practice? This is the biggest concern in this scenario, as it may indicate inadequate veterinary care and pain management for more GEU animals than just Boyd's monkeys. The IACUC has an opportunity to use this situation to improve pain management and veterinary care for all of the university's animals.

\section{Larry Carbone}

Private Consultant, San Francisco, USA.

e-mail: larrycarbonedvm@gmail.com

Published online: 18 December 2019

https://doi.org/10.1038/s41684-019-0442-3

\section{References}

1. Institute for Laboratory Animal Research. Guide for the Care and Use of Laboratory Animals, 8th ed. (National Academies Press, Washington D.C., 2011).

2. American College of Veterinary Anesthesia and Analgesia. American College of Veterinary Anesthesiologists' position paper on the treatment of pain in animals. 2006. http://acvaa.org/docs/ Pain_Treatment Accessed September 15, 2019.

3. Kohn, D., Martin, T. E., Foley, P. E., Morris, T. H., Swindle, M. M. Vogler, G. A. \& Wixson, S. K. JAALAS 46(2), 97-108(12) (2007).

4. Carbone, L. Pain Management Standards in the Eighth Edition of the Guide for the Care and Use of Laboratory Animals. JAALAS 51(3), 322-328 (2012).

5. Beverly, A., Kaye, A. D., Ljungqvist, O. \& Urman, O. Anesthesiology Clinics 35(2), el15-e143 (2017).

6. Animal Welfare Act Regulations. 7 U.S.C. 2131-2159; 7 CFR 2.22, 2.80 , and 371.7

\section{Acknowledgements}

The author thanks David Takacs, Alicia Karas, and Jeremy Lieberman for helpful input on this essay. 